\title{
A GENERALIZATION OF THE MATRIX FORM OF THE BRUNN-MINKOWSKI INEQUALITY
}

\author{
JUN YUAN and GANGSONG LENG
}

(Received 4 March 2005; revised 24 March 2006)

Communicated by A. Rubinov

\begin{abstract}
In this paper, we establish an extension of the matrix form of the Brunn-Minkowski inequality. As applications, we give generalizations on the metric addition inequality of Alexander.

2000 Mathematics subject classification: primary 52A40.

Keywords and phrases: Brunn-Minkowski inequality, metric addition inequality, Ky Fan inequality, Bellman's inequality, matrix, simplex.
\end{abstract}

\section{Introduction}

The Brunn-Minkowski inequality is one of the most important geometric inequalities. There is a vast amount of work on its generalizations and on its connections with other areas, (see $[2,5-13,21,22]$ ). An excellent survey on this inequality is provided by Gardner (see [12]). The matrix form of the Brunn-Minkowski inequality (see $[14,15]$ ) asserts that if $A$ and $B$ are two positive definite matrices of order $n$, then

$$
|A+B|^{1 / n} \geq|A|^{1 / n}+|B|^{1 / n},
$$

with equality if and only if $A=c B(c \geq 0)$, where $|A|$ denotes the determinant of $A$.

In [4], Bergström proved the following interesting inequality, which is analogous to (1.1).

If $A$ and $B$ are positive definite matrices of order $n$, and $A_{(i)}, B_{(i)}$ denote the sub-matrices obtained by deleting the $i$-th row and column, then

$$
\frac{|A+B|}{\left|A_{(i)}+B_{(i)}\right|} \geq \frac{|A|}{\left|A_{(i)}\right|}+\frac{|B|}{\left|B_{(i)}\right|} \text {. }
$$

Supported in part by the National Natural Science Foundation of China (Grant No. 10671117).

(C) 2007 Australian Mathematical Society 1446-7887/07 \$A2.00+0.00 
In [9], Ky Fan gave a simultaneous generalization of (1.1) and (1.2). He established the following elegant inequality.

Let $A_{k}$ denote the principal sub-matrix of $A$ formed by taking the first $k$ rows and columns of $A$. If $C=A+B$, where $A$ and $B$ are positive definite matrices of order $n$, then

$$
\left(\frac{|C|}{\left|C_{k}\right|}\right)^{1 /(n-k)} \geq\left(\frac{|A|}{\left|A_{k}\right|}\right)^{1 /(n-k)}+\left(\frac{|B|}{\left|B_{k}\right|}\right)^{1 /(n-k)}
$$

In this paper, a new generalization of the matrix form of the Brunn-Minkowski inequality is presented, which is an extension of (1.3) also.

Let $I_{n-k}$ denote the unit matrix of order $n-k,(0 \leq k<n)$. One of our main results is the following theorem.

THEOREM 1.1. Let $A$ and $B$ be positive definite matrices of order $n$, and let $a$ and $b$ be two nonnegative real numbers such that $A>a I_{n}$ and $B>b I_{n}$. If $C=A+B$, then

$$
\begin{aligned}
& \left(\frac{|C|}{\left|C_{k}\right|}-\left|(a+b) I_{n-k}\right|\right)^{1 /(n-k)} \\
& \quad \geq\left(\frac{|A|}{\left|A_{k}\right|}-\left|a I_{n-k}\right|\right)^{1 /(n-k)}+\left(\frac{|B|}{\left|B_{k}\right|}-\left|b I_{n-k}\right|\right)^{1 /(n-k)}
\end{aligned}
$$

with equality if and only if $a^{-1} A=b^{-1} B$.

The other aim of this paper is to provide a generalization of the metric addition inequality of Alexander. The concept of metric addition began with Oppenheim in [20], and was first explicitly defined and named by Alexander in [1].

Let $\Omega_{1}=\left\{P_{0}^{(1)}, \ldots, P_{n}^{(1)}\right\}$ and $\Omega_{2}=\left\{P_{0}^{(2)}, \ldots, P_{n}^{(2)}\right\}$ denote two simplices in the $n$-dimensional Euclidean space $R^{n}$ with vertices $P_{0}^{(1)}, \ldots, P_{n}^{(1)}$ and $P_{0}^{(2)}, \ldots, P_{n}^{(2)}$, respectively. If there exists a set of points $\Omega_{3}=\left\{P_{0}^{(3)}, \ldots, P_{n}^{(3)}\right\}$, such that

$$
\left|P_{i}^{(3)}-P_{j}^{(3)}\right|^{2}=\left|P_{i}^{(1)}-P_{j}^{(1)}\right|^{2}+\left|P_{i}^{(2)}-P_{j}^{(2)}\right|^{2},
$$

then $\Omega_{3}$ is called metric addition of $\Omega_{1}$ and $\Omega_{2}$, and is denoted by

$$
\Omega_{3}=\Omega_{1}+\Omega_{2}
$$

It can be proved that the set of points $\Omega_{3}$ exists and is an $n$-dimensional simplex (see [1]). Alexander conjectured the following inequality:

$$
V^{2}\left(\Omega_{3}\right) \geq V^{2}\left(\Omega_{1}\right)+V^{2}\left(\Omega_{2}\right) .
$$


However in [23], Yang and Zhang proved that (1.7) is not true, and gave the following correct form

$$
V^{2 / n}\left(\Omega_{3}\right) \geq V^{2 / n}\left(\Omega_{1}\right)+V^{2 / n}\left(\Omega_{2}\right),
$$

with equality if and only if $\Omega_{1}$ and $\Omega_{2}$ are similar.

As an application of Theorem 1.1, we establish the following theorem, which is a special case of Theorem 4.1 of this paper.

THEOREM 1.2. Let simplex $\Omega_{3}$ be a metric addition of simplex $\Omega_{1}$ and simplex $\Omega_{2}$. Let $D_{1}$ and $D_{2}$ be compact domains in $R^{n}$ and $D_{1} \subset \Omega_{1}, D_{2} \subset \Omega_{2}$. Then

$$
\begin{aligned}
& {\left[V^{2}\left(\Omega_{3}\right)-\left(V^{2 / n}\left(D_{1}\right)+V^{2 / n}\left(D_{2}\right)\right)^{n}\right]^{1 / n}} \\
& \quad \geq\left[V^{2}\left(\Omega_{1}\right)-V^{2}\left(D_{1}\right)\right]^{1 / n}+\left[V^{2}\left(\Omega_{2}\right)-V^{2}\left(D_{2}\right)\right]^{1 / n} .
\end{aligned}
$$

The equality holds if and only if $\Omega_{1}$ and $\Omega_{2}$ are similar and $\left(V\left(\Omega_{1}\right), V\left(\Omega_{2}\right)\right)=$ $\mu\left(V\left(D_{1}\right), V\left(D_{2}\right)\right)$, where $\mu$ is a constant.

REMARK 1.3. Taking $D_{1}=D_{2}=\varnothing$ or taking $D_{1}=\Omega_{1}, D_{2}=\Omega_{2}$ in Theorem 1.2, we can obtain (1.8). Hence (1.9) is a generalization of (1.8).

\section{Definitions and lemmas}

Let $S_{n}(R)$ denote the set of $n \times n$ real symmetric matrices. Let $I_{n}$ denote the $n \times n$ unit matrix. We use the notation $A>0(A \geq 0)$ if $A$ is a positive definite (positive semi-definite) matrix, and $A^{T}$ denotes the transpose of $A$. Let $A, B \in S_{n}(R)$. Then $A>B(A \geq B)$ if and only if $A-B>0(A-B \geq 0)$. Let $k_{n}$ denote the volume of the unit ball in $R^{n}$.

DEFINITION 2.1. Let $A=\left[\begin{array}{cc}A_{k} & A_{12} \\ A_{21} & A_{22}\end{array}\right]$ be a matrix of order $n$, and let $A_{k}$ denote the principal sub-matrix of $A$ formed by taking the first $k$ rows and columns of $A$. If $A_{k}$ is nonsingular, then $A_{22}-A_{21} A_{k}^{-1} A_{12}$ is called a Schur complement of $A$, with respect to $A_{k}$, which is denoted by $A / A_{k}$.

Obviously, if $A_{k}$ is a matrix of order 0 , then $A / A_{k}=A$.

LEMMA 2.2. Let $A \in S_{n}(R), A>0$, and $A_{k}$ be its $k$-th order principal minor. Then

$$
A / A_{k}>0 \text { and }\left|A / A_{k}\right|=\frac{|A|}{\left|A_{k}\right|} \text {. }
$$

The proof of Lemma 2.2 can be found in [17, page 22]. 
LEMMA 2.3 ([10,16]). Let $A, B \in S_{n}(R), A>0, B>0$, and $A_{k}$ and $B_{k}$ be $k$-th order principal minors of $A$ and $B$, respectively. Then

$$
(A+B) /\left(A_{k}+B_{k}\right) \geq A / A_{k}+B / B_{k} .
$$

LEMMA 2.4. Let $A, B \in S_{n}(R), A>B>0$. Then

$$
|A|>|B|
$$

The proof of Lemma 2.4 can be found in [17, page 472].

LEMMA 2.5 ([19]). Let $A, B \in S_{n}(R), A \geq B>0, A_{k}, B_{k}$ be $k$-th order principal minors of $A$ and $B$, respectively. Then

$$
\left|A / A_{k}\right| \geq\left|B / B_{k}\right|
$$

LEMMA 2.6. Let $A, B \in S_{n}(R), A>0, B>0$. Then there exists an invertible matrix $P$ satisfying $\left|P^{T} P\right|=1$ such that $P^{T} A P=\operatorname{diag}\left(a_{1}, \ldots, a_{n}\right)$ and $P^{T} B P=$ $\operatorname{diag}\left(b_{1}, \ldots, b_{n}\right)$.

LEMMA 2.7. Let $x_{i} \geq 0, y_{i} \geq 0(i=1, \ldots, n)$. Then

$$
\left(\prod_{i=1}^{n} x_{i}\right)^{1 / n}+\left(\prod_{i=1}^{n} y_{i}\right)^{1 / n} \leq\left(\prod_{i=1}^{n}\left(x_{i}+y_{i}\right)\right)^{1 / n}
$$

with equality if and only if $x_{i}=v y_{i}$, where $v$ is a constant.

This is a special case of Maclaurin's inequality.

LEMMA 2.8 (Bellman's inequality). Suppose that $a=\left\{a_{1}, \ldots, a_{n}\right\}$ and $b=$ $\left\{b_{1}, \ldots, b_{n}\right\}$ are two $n$-tuples of positive real numbers, and $p>1$ such that

$$
a_{1}^{p}-\sum_{i=2}^{n} a_{i}^{p}>0 \text { and } b_{1}^{p}-\sum_{i=2}^{n} b_{i}^{p}>0 .
$$

Then

$$
\left(\left(a_{1}+b_{1}\right)^{p}-\sum_{i=2}^{n}\left(a_{i}+b_{i}\right)^{p}\right)^{1 / p} \geq\left(a_{1}^{p}-\sum_{i=2}^{n} a_{i}^{p}\right)^{1 / p}+\left(b_{1}^{p}-\sum_{i=2}^{n} b_{i}^{p}\right)^{1 / p}
$$

with equality if and only if $a=v b$, where $v$ is a constant.

The proof of Lemma 2.8 can be found in [3, page 38]. 


\section{Proof of Theorem 1.1}

PROOF. According to Lemma 2.2 and Lemma 2.3, we have

and

$$
\left|A / A_{k}\right|=\frac{|A|}{\left|A_{k}\right|}, \quad\left|B / B_{k}\right|=\frac{|B|}{\left|B_{k}\right|},
$$

$$
\frac{|A+B|}{\left|A_{k}+B_{k}\right|}=\left|(A+B) /\left(A_{k}+B_{k}\right)\right| \geq\left|\left(A / A_{k}\right)+\left(B / B_{k}\right)\right| \text {. }
$$

So

$$
\begin{aligned}
& \left(\frac{|A+B|}{\left|A_{k}+B_{k}\right|}-\left|(a+b) I_{n-k}\right|\right)^{1 /(n-k)} \\
& \quad \geq\left(\left|\left(A / A_{k}\right)+\left(B / B_{k}\right)\right|-\left|(a+b) I_{n-k}\right|\right)^{1 /(n-k)} .
\end{aligned}
$$

Let $\widetilde{A}=A / A_{k}>0$ and $\widetilde{B}=B / B_{k}>0$. Then to prove (1.4), we need only to prove the following inequality

$$
\begin{aligned}
& \left(|\tilde{A}+\widetilde{B}|-\left|(a+b) I_{n-k}\right|\right)^{1 /(n-k)} \\
& \quad \geq\left(|\widetilde{A}|-\left|a I_{n-k}\right|\right)^{1 /(n-k)}+\left(|\widetilde{B}|-\left|b I_{n-k}\right|\right)^{1 /(n-k)} .
\end{aligned}
$$

Notice that $\widetilde{A}$ and $\widetilde{B}$ are matrices of order $n-k$. By condition $A>a I_{n}, B>b I_{n}$ and Lemma 2.5, we have $\widetilde{A}>a I_{n-k}, \widetilde{B}>b I_{n-k}$. By Lemma 2.6, there is an invertible matrix $P$ such that $\left|P^{T} P\right|=1$, and

So

$$
P^{T} \widetilde{A} P=\operatorname{diag}\left(a_{1}, \ldots, a_{n-k}\right), \quad P^{T} \widetilde{B} P=\operatorname{diag}\left(b_{1}, \ldots, b_{n-k}\right) .
$$

$$
|\widetilde{A}|=\left|P^{T} \widetilde{A} P\right|=\prod_{i=1}^{n-k} a_{i}, \quad|\widetilde{B}|=\left|P^{T} \widetilde{B} P\right|=\prod_{i=1}^{n-k} b_{i}, \quad \text { and } \quad|\widetilde{A}+\widetilde{B}|=\prod_{i=1}^{n-k}\left(a_{i}+b_{i}\right) .
$$

It is straightforward to see that (3.3) holds if and only if

$$
\begin{aligned}
& \left(\prod_{i=1}^{n-k}\left(a_{i}+b_{i}\right)-(a+b)^{n-k}\right)^{1 /(n-k)} \\
& \quad \geq\left(\prod_{i=1}^{n-k} a_{i}-a^{n-k}\right)^{1 /(n-k)}+\left(\prod_{i=1}^{n-k} b_{i}-b^{n-k}\right)^{1 /(n-k)}
\end{aligned}
$$

Now we prove (3.4). Put $X^{n-k}=\prod_{i=1}^{n-k} a_{i}-a^{n-k}$ and $Y^{n-k}=\prod_{i=1}^{n-k} b_{i}-b^{n-k}$. Then

$$
X^{n-k}+a^{n-k}=\prod_{i=1}^{n-k} a_{i}, \quad Y^{n-k}+b^{n-k}=\prod_{i=1}^{n-k} b_{i} .
$$


Applying Minkowski inequality, we have

$$
\begin{aligned}
\left((X+Y)^{n-k}+(a+b)^{n-k}\right)^{1 /(n-k)} & \leq\left(X^{n-k}+a^{n-k}\right)^{1 /(n-k)}+\left(Y^{n-k}+b^{n-k}\right)^{1 /(n-k)} \\
& =\left(\prod_{i=1}^{n-k} a_{i}\right)^{1 /(n-k)}+\left(\prod_{i=1}^{n-k} b_{i}\right)^{1 /(n-k)}
\end{aligned}
$$

Applying Lemma 2.7 to the right of the above inequality, we obtain

$$
\left((X+Y)^{n-k}+(a+b)^{n-k}\right)^{1 /(n-k)} \leq\left(\prod_{i=1}^{n-k}\left(a_{i}+b_{i}\right)\right)^{1 /(n-k)},
$$

which implies that $(X+Y)^{n-k} \leq \prod_{i=1}^{n-k}\left(a_{i}+b_{i}\right)-(a+b)^{n-k}$. It follows that

$$
X+Y \leq\left(\prod_{i=1}^{n-k}\left(a_{i}+b_{i}\right)-(a+b)^{n-k}\right)^{1 /(n-k)},
$$

which is just inequality (3.4).

REMARK 3.1. Let $a=b=0$ in Theorem 1.1. Then we get the Ky Fan inequality (1.3). Let $k=0$ in Theorem 1.1, and we obtain

$$
\left(|A+B|-\left|(a+b) I_{n}\right|\right)^{1 / n} \geq\left(|A|-\left|a I_{n}\right|\right)^{1 / n}+\left(|B|-\left|b I_{n}\right|\right)^{1 / n},
$$

with equality if and only if $a^{-1} A=b^{-1} B$.

This is [18, Equation (23)], so Theorem 1.1 is a generalization of the Ky Fan inequality (1.3) and (3.5).

Replacing $A$ and $B$ by $\lambda A$ and $\mu B$, and at the same time replacing $a$ and $b$ by $\lambda a$ and $\mu b$ in Theorem 1.1, yields the following corollary.

COROLlARY 3.2. Let $A, B \in S_{n}(R)$, and $A_{k}$ and $B_{k}$ be $k$-th order principal minors of $A$ and $B$ respectively. Let $C=\lambda A+\mu B, a \geq 0, b \geq 0$. If $A>a I_{n}, B>b I_{n}$, then

$$
\begin{aligned}
& \left(\frac{|C|}{\left|C_{k}\right|}-\left|(\lambda a+\mu b) I_{n-k}\right|\right)^{1 /(n-k)} \\
& \geq \lambda\left(\frac{|A|}{\left|A_{k}\right|}-\left|a I_{n-k}\right|\right)^{1 /(n-k)}+\mu\left(\frac{|B|}{\left|B_{k}\right|}-\left|b I_{n-k}\right|\right)^{1 /(n-k)}
\end{aligned}
$$

for all $\lambda>0, \mu>0$, with equality if and only if $a^{-1} A=b^{-1} B$.

By induction, we infer the following. 
COROLlarY 3.3. Let $A_{i} \in S_{n}(R), a_{i} \geq 0, \lambda_{i}>0, A_{i}>a_{i} I_{n}$, and $A_{i(k)}$ be $k$-th order principal minors of $A_{i}, i=1, \ldots, m$. Then

$$
\left(\frac{\left|\sum_{i=1}^{m} \lambda_{i} A_{i}\right|}{\left|\sum_{i=1}^{m} \lambda_{i} A_{i(k)}\right|}-\left|\sum_{i=1}^{m} \lambda_{i} a_{i} I_{n-k}\right|\right)^{1 /(n-k)} \geq \sum_{i=1}^{m} \lambda_{i}\left(\frac{\left|A_{i}\right|}{\left|A_{i(k)}\right|}-\left|a_{i} I_{n-k}\right|\right)^{1 /(n-k)},
$$

with equality if and only if $a_{1}^{-1} A_{1}=\cdots=a_{m}^{-1} A_{m}$.

Applying the generalized arithmetic-geometric mean inequality to the right side of (3.7), we get the following inequality.

CoRollary 3.4. Let $A_{i} \in S_{n}(R), a_{i} \geq 0, \lambda_{i}>0$, and $\sum_{i=1}^{m} \lambda_{i}=1, A_{i}>a_{i} I_{n}$, and $A_{i(k)}$ be $k$-th order principal minors of $A_{i}, i=1, \ldots, m$. Then

$$
\frac{\left|\sum_{i=1}^{m} \lambda_{i} A_{i}\right|}{\left|\sum_{i=1}^{m} \lambda_{i} A_{i(k)}\right|}-\left|\sum_{i=1}^{m} \lambda_{i} a_{i} I_{n-k}\right| \geq \prod_{i=1}^{m}\left(\frac{\left|A_{i}\right|}{\left|A_{i(k)}\right|}-\left|a_{i} I_{n-k}\right|\right)^{\lambda_{i}} \text {. }
$$

When $a_{1}=\cdots=a_{m}=0$, the equality holds in (3.8) if and only if $A_{1}, \ldots, A_{m}$ are equal.

Taking $i=2, k=0$ in Corollary 3.4, we obtain a generalization of the Ky Fan concave theorem as follows.

CoROllary 3.5. Let $A_{i} \in S_{n}(R), a_{i} \geq 0, A_{i}>a_{i} I_{n}(i=1,2)$. Then

$$
\left|\lambda A_{1}+(1-\lambda) A_{2}\right|-\left[\lambda a_{1}+(1-\lambda) a_{2}\right]^{n} \geq\left(\left|A_{1}\right|-a_{1}^{n}\right)^{\lambda}\left(\left|A_{2}\right|-a_{2}^{n}\right)^{1-\lambda},
$$

where $0 \leq \lambda \leq 1$.

\section{Inequalities for metric addition}

Let $\Omega_{l}=\left\{P_{0}^{(l)}, \ldots, P_{n}^{(l)}\right\}(1 \leq l \leq m)$ be a simplex in $R^{n}$. For any $\lambda_{l}>0$ $(1 \leq l \leq m)$, there exists a unique simplex $\Omega_{m+1}=\left\{P_{0}^{(m+1)}, \ldots, P_{n}^{(m+1)}\right\}$ such that

$$
\left|P_{i}^{(m+1)}-P_{j}^{(m+1)}\right|^{2}=\sum_{l=1}^{m} \lambda_{l}\left|P_{i}^{(l)}-P_{j}^{(l)}\right|^{2} .
$$

Then $\Omega_{m+1}$ is called the weighted metric addition of $\Omega_{1}, \ldots, \Omega_{m}$, denoted by

$$
\Omega_{m+1}=\sum_{l=1}^{m} \lambda_{l} \Omega_{l}
$$

(see $[1,23])$. On the weighted metric addition, we have the following theorem. 
THEOREM 4.1. Let $\Omega_{i}$ be an n-dimensional simplex in $R^{n}(1 \leq i \leq m)$. If $\Omega_{m+1}=\sum_{i=1}^{m} \lambda_{i} \Omega_{i}$ and compact domains $D_{i} \subset \Omega_{i}$, then

$$
\left.\left.\left[V^{2}\left(\Omega_{m+1}\right)\right)-\left(\sum_{i=1}^{m} \lambda_{i} V^{2 / n}\left(D_{i}\right)\right)^{n}\right]^{1 / n} \geq \sum_{i=1}^{m} \lambda_{i}\left[V^{2}\left(\Omega_{i}\right)\right)-V^{2}\left(D_{i}\right)\right]^{1 / n}
$$

The equality holds if and only if $\Omega_{1}, \ldots, \Omega_{m}$ are similar and $\left(V\left(\Omega_{1}\right), \ldots, V\left(\Omega_{m}\right)\right)$ $=\mu\left(V\left(D_{1}\right), \ldots, V\left(D_{m}\right)\right)$, where $\mu$ is a constant.

PROOF. Let $a_{i j}^{(l)}$ be the distance between $P_{i}^{(l)}$ and $P_{j}^{(l)}$. Let

$$
\rho_{i j}^{(l)}=\left(a_{i 0}^{(l)}\right)^{2}+\left(a_{0 j}^{(l)}\right)^{2}-\left(a_{i j}^{(l)}\right)^{2}, \quad(0 \leq i, j \leq n) .
$$

Then the matrix $A^{(l)}=\left(\rho_{i j}^{(l)}\right)_{n \times n}$ is a positive definite matrix.

It is straightforward to verify that

$$
A^{(m+1)}=\sum_{i=1}^{m} \lambda_{i} A^{(i)}
$$

then by the volume formula of a simplex, we have

$$
\left|A^{(i)}\right|=2^{n} n !^{2} V^{2}\left(\Omega_{i}\right),
$$

where $1 \leq i \leq m+1$.

Let

$$
a_{i}^{n}=2^{n} n !^{2} V^{2}\left(D_{i}\right), \quad(1 \leq i \leq m) .
$$

Since $D_{i} \subset \Omega_{i}, 1 \leq i \leq m$, then $\left|A_{i}\right|>a_{i}^{n}, 1 \leq i \leq m$. Setting $k=0$ in Corollary 3.3, we have

$$
\left[\left|\sum_{i=1}^{m} \lambda_{i} A_{i}\right|-\left(\sum_{i=1}^{m} \lambda_{i} a_{i}\right)^{n}\right]^{1 / n} \geq \sum_{i=1}^{m} \lambda_{i}\left(\left|A_{i}\right|-a_{i}^{n}\right)^{1 / n},
$$

with equality if and only if $a_{1}^{-1} A^{(1)}=\cdots=a_{m}^{-1} A^{(m)}$.

By (4.4), we have

$$
\left[\left|A^{(m+1)}\right|-\left(\sum_{i=1}^{m} \lambda_{i} a_{i}\right)^{n}\right]^{1 / n} \geq \sum_{i=1}^{m} \lambda_{i}\left(\left|A^{(i)}\right|-a_{i}^{n}\right)^{1 / n} .
$$

Substituting (4.5) and (4.6) into (4.7) and rearranging, we obtain (4.3). 
Let $D_{1}$ and $D_{2}$ be two closed balls with radii $r_{1}$ and $r_{2}$, respectively. We infer the following.

COROLLARY 4.2. Let $\Omega_{3}=\Omega_{1}+\Omega_{2}$, and $r\left(\Omega_{1}\right)$ and $r\left(\Omega_{2}\right)$ be the radii of simplex $\Omega_{1}$ and $\Omega_{2}$, respectively. If $0 \leq r_{1} \leq r\left(\Omega_{1}\right), 0 \leq r_{2} \leq r\left(\Omega_{2}\right)$, then

$$
\left(V^{2}\left(\Omega_{3}\right)-\left(r_{1}^{2}+r_{2}^{2}\right)^{n} k_{n}^{2}\right)^{1 / n} \geq\left(V^{2}\left(\Omega_{1}\right)-r_{1}^{2 n} k_{n}^{2}\right)^{1 / n}+\left(V^{2}\left(\Omega_{2}\right)-r_{2}^{2 n} k_{n}^{2}\right)^{1 / n}
$$

When $r_{1}=r_{2}=0$, there is equality if and only if $\Omega_{1}$ and $\Omega_{2}$ are similar; when $r_{1} \neq 0$ and $r_{2} \neq 0$, equality holds if and only if $\Omega_{1}$ and $\Omega_{2}$ are similar and $r_{1}^{n} / r_{2}^{n}=$ $V\left(\Omega_{1}\right) / V\left(\Omega_{2}\right)$.

ProOF. From (1.8) and applying Bellman's inequality (2.6), we have

$$
\begin{aligned}
\left(V^{2}\left(\Omega_{3}\right)-\left(r_{1}^{2}+r_{2}^{2}\right)^{n} k_{n}^{2}\right)^{1 / n} & \geq\left(\left(V^{2 / n}\left(\Omega_{1}\right)+V^{2 / n}\left(\Omega_{2}\right)\right)^{n}-\left(r_{1}^{2}+r_{2}^{2}\right)^{n} k_{n}^{2}\right)^{1 / n} \\
& \geq\left(V^{2}\left(\Omega_{1}\right)-r_{1}^{2 n} k_{n}^{2}\right)^{1 / n}+\left(V^{2}\left(\Omega_{2}\right)-r_{2}^{2 n} k_{n}^{2}\right)^{1 / n}
\end{aligned}
$$

The proof is complete.

\section{Acknowledgment}

The authors are most grateful to the referee for his valuable suggestions.

\section{References}

[1] R. Alexander, The geometry of metric and linear space (Springer-Verlag, Berlin, 1975) pp. 57-65.

[2] I. J. Bakelman, Convex analysis and nonlinear geometric elliptic equations (Springer, Berlin, 1994).

[3] E. F. Beckenbach and R. Bellman, Inequalities (Springer, Berlin, 1961).

[4] H. Bergström, A triangle inequality for matrices (Den Elfte Skandiaviski Matematiker-kongress, Trondheim, 1949).

[5] C. Borell, 'The Brunn-Minkowski inequality in Gauss space', Invent Math. 30 (1975), 202-216.

[6] _ 'Capacitary inequality of the Brunn-Minkowski inequality type', Math. Ann. 263 (1993), 179-184.

[7] Y. D. Burago and V. A. Zalgaller, Geometric inequalities, (Translated from Russian: Springer Series in Soviet Mathematics) (Springer, New York, 1988).

[8] K. Fan, 'Problem 4786', Amer. Math. Monthly 65 (1958), 289.

[9] - 'Some inequalities concerning positive-definite Hermitian matrices', Proc. Cambridge Phil. Soc. 51 (1958), 414-421.

[10] M. Fiedler and T. Markham, 'Some results on the Bergström and Minkowski inequalities', Linear Algebra Appl. 232 (1996), 199-211. 
[11] R. J. Gardner, Geometric tomography, Encyclopædia of Mathematics and its Applications 58 (Cambridge University Press, Cambridge, 1995).

[12] — 'The Brunn-Minkowski inequality', Bull. Amer. Math. Soc. (N.S.) 39 (2002), 355-405.

[13] R. J. Gardner and P. Gronchi, 'A Brunn-Minkowski inequality for the integer lattice', Trans. Amer. Math. Soc. 353 (2001), 3995-4024.

[14] E. V. Haynesworth, 'Note on bounds for certain determinants', Duke Math. J. 24 (1957), 313-320.

[15] _ 'Bounds for determinants with positive diagonals', Trans. Amer. Math. Soc. 96 (1960), $395-413$.

[16] E. V. Haynsworth, 'Applications of an inequality for the Schur complement', Proc. Amer. Math. Soc. 24 (1970), 512-516.

[17] R. Horn and C. R. Johnson, Matrix analysis (Cambridge University Press, Cambridge, 1985).

[18] G. S. Leng, 'The Brunn-Minkowski inequality for volume differences', Adv. in Appl. Math. 32 (2004), 615-624.

[19] C. K. Li and R. Mathias, 'Extremal characterizations of Schur complement and resulting inequalities', SIAM Rev. 42 (2000), 233-246.

[20] A. Oppenheim, 'Advanced problems 5092', Amer. Math. Monthly 701 (1963), 444.

[21] R. Osserman, 'The Brunn-Minkowski inequality for multiplictities', Invent. Math. 125 (1996), 405-411.

[22] R. Schneider, Convex bodies: The Brunn-Minkowski theory (Cambridge University Press, Cambridge, 1993).

[23] L. Yang and J. Z. Zhang, 'On Alexander's conjecture', Chinese Sci. Bull. 27 (1982), 1-3.

School of Mathematics and Computer Science

Nanjing Normal University

Nanjing 210097

P.R. China

e-mail: yuanjun@graduate.shu.edu.cn
Department of Mathematics

Shanghai University

Shanghai 200444

P. R. China

e-mail: gleng@staff.shu.edu.cn 